\title{
Programa de Formación en Salud Pública para Trabajadores de Salud en la Frontera Brasil-Uruguay
}

\author{
Frederico Peres, ${ }^{1}$ Maria Pasionaria Blanco Centurión, ${ }^{1}$ Juliana Monteiro Bastos da Silva, ${ }^{1}$ \\ Ana Laura Brandão, ${ }^{1}$ José Fernando de Souza Verani, ${ }^{1}$ Marisa Buglioli, ${ }^{2}$ Elizabeth Ortega ${ }^{3}$ \\ y Adriana Brescia ${ }^{4}$
}

Forma de citar

Peres F, Blanco Centurión MP, Silva JMB, Brandão AL, Verani JFS, Buglioli M, et al. Programa de Formación en Salud Pública para Trabajadores de Salud en la Frontera Brasil-Uruguay. Rev Panam Salud Publica. 2020;44:e95. https://doi.org/10.26633/ RPSP.2020.95

RESUMEN

El principal objetivo del Programa de Formación en Salud Pública para la Frontera Brasil-Uruguay fue fortalecer las capacidades formativas locales en salud pública a través del desarrollo de habilidades y competencias junto a trabajadores de salud de cuatro zonas fronterizas. El diseño metodológico del Programa, basado en talleres docentes con aplicación de metodologías activas de la formación en salud y mediados por tecnologías educacionales, favoreció la organización de un espacio dialógico permanente entre docentes, gestores y actores de la práctica de ambos países que, entre 2017 y 2018, resultó en: a) el desarrollo de capacidades locales para el mapeo de los principales problemas de vigilancia de la salud en las cuatro zonas fronterizas participantes; b) la discusión de los límites y perspectivas de acción de los servicios de salud existentes en ambos países, dentro de los principios y lineamientos del campo de la salud pública; c) la apropiación de herramientas y procesos pedagógicos que permitieron la incorporación del aspecto de salud pública y de la vigilancia de la salud en los diferentes procesos y espacios organizativos del sector salud local; y d) la construcción de una capacidad permanente para el desarrollo de habilidades y competencias junto a trabajadores del sector salud, en una lógica de educación permanente en salud. Los logros del Programa y sus contribuciones a los servicios de salud locales lo habilitan, de manera efectiva, como acción estratégica para el fortalecimiento de capacidades formativas y de investigación en la frontera Brasil-Uruguay.

Palabras clave Desarrollo de personal; aprendizaje basado en problemas; educación en salud pública profesional; vigilancia en salud pública; salud fronteriza; Brasil; Uruguay.

La formación y el desarrollo de recursos humanos en salud (RRHHS) son acciones estratégicas para el fortalecimiento de los sistemas, programas y servicios de salud, principalmente en los países en desarrollo que todavía enfrentan déficits en la provisión y distribución de la fuerza de trabajo en el sector salud y los efectos de las transiciones demográfica y sanitaria determinadas por los procesos de desarrollo económico y social (1-3).

En América Latina, región caracterizada por la dinámica de los procesos de desarrollo que vivencian la mayoría de sus países, a pesar de los avances en términos del desarrollo social y económico y de la estructuración de los sistemas de salud nacionales todavía persisten profundos desafíos con relación al acceso a los servicios de salud y a las iniquidades vivenciadas principalmente por grupos e individuos en situación de vulnerabilidad $(3,4)$. A estos desafíos se agregan la inequidad de la distribución de los profesionales de salud entre los países de la región y en cada país, la limitada disponibilidad de ofertas formativas que consideren el enfoque cultural y el

\footnotetext{
1 Fundación Oswaldo Cruz (ENSP/Fiocruz), Rio de Janeiro, Brasil $₫$ Frederico Peres, frederico.peres@fiocruz.br

2 Universidad de la República, Montevideo, Uruguay
} 
desarrollo de competencias armonizadas con las necesidades de los sistemas de salud, y el perfil de la fuerza de trabajo regional en lo que se refiere a las demandas de los grupos e individuos a quienes sirven (3-5).

Las zonas fronterizas, en general, constituyen unidades de elevado interés sanitario y, también, espacios que representan grandes desafíos a la formación, distribución y retención de trabajadores de salud (6), donde los problemas de salud requieren la realización conjunta de actividades para lograr el control efectivo de enfermedades, la garantía del acceso a la atención a la salud y la promoción de la salud en el territorio (7).

La frontera entre Brasil y Uruguay es un rico territorio para la comprensión de las dinámicas de intercambio fronterizo entre diferentes servicios, programas y sistemas de salud, que ha sido explorado por diversos autores (6-12). Considerando la extensión del Brasil y todos los países con los que limita, la frontera con Uruguay es la más abierta, densa y homogéneamente poblada $(12,13)$; es, asimismo, una de las más integradas, con la presencia de varias ciudades gemelas y fuerte interacción $(12,14)$.

En general, los municipios ubicados en la frontera Brasil-Uruguay han tenido grandes dificultades para proporcionar atención integral a la salud en el contexto binacional, como la falta de recursos humanos especializados y la distancia entre los municipios y los centros de referencia (14-16).

Dada esta realidad, la construcción y oferta de estrategias de formación para la fuerza de trabajo que actúa en la frontera Brasil-Uruguay necesita considerar la dinámica de organización y prestación de servicios de salud en ambos países, proporcionando elementos que puedan mejorar las prácticas profesionales, el conocimiento de realidades de los dos sistemas de salud, la generación de datos e indicadores para la toma de decisiones y el fortalecimiento de las capacidades locales, en particular las capacidades formativas y de investigación (17-20).

En este artículo se presentan los principales resultados del Programa de Formación en Salud Pública para la Frontera Brasil-Uruguay, ofrecido a trabajadores de salud que actúan en distintos municipios y departamentos de esta zona fronteriza. El principal objetivo del Programa fue fortalecer las capacidades formativas en salud pública en la zona de frontera, en una perspectiva de formación de activadores de la educación permanente en salud. Los objetivos secundarios incluyen el desarrollo de habilidades y competencias junto a los educandos (trabajadores del sector salud local), que les permita realizar análisis comparados sobre las políticas, programas y sistemas de salud de ambos los países, el uso de técnicas y herramientas de análisis de datos de salud en nivel local y el desarrollo de estrategias formativas basadas en metodologías activas de la educación permanente en salud.

\section{DISEÑO DEL PROGRAMA DE FORMACIÓN}

El Programa se inserta en el marco de un acuerdo de cooperación trilateral entre los Ministerios de Salud de Brasil y de Uruguay, con recursos del Banco de Desarrollo Alemán (21). Este acuerdo de cooperación priorizó el fortalecimiento de la infraestructura del sector salud en municipios fronterizos con menos de 5000 habitantes, localizados en los departamentos de Cerro Largo, Rivera, Artigas y Rocha, en Uruguay y, como contraparte técnica, demandaba la capacitación de profesionales de salud de estos municipios, acción estratégica que fue delegada a la Escuela Nacional de Salud Pública Sergio Arouca de la Fundación Oswaldo Cruz (ENSP / Fiocruz) del Ministerio de Salud de Brasil.

\section{Localización geográfica}

La frontera entre Brasil y Uruguay fue delimitada por los tratados de 1851 y 1909 y la línea divisoria entre los países se extiende por $1069 \mathrm{~km}$, a lo largo de $749 \mathrm{~km}$ de ríos, canales y lagunas y $320 \mathrm{~km}$ de frontera seca (13). En Brasil, 11 municipios limitan con Uruguay: Chuí, Santa Vitória do Palmar, Jaguarão, Erval, Aceguá, Bagé, Dom Pedrito, Santana do Livramento, Quaraí, Uruguaiana y Barra do Quaraí. En Uruguay, a su vez, existen cinco departamentos que hacen frontera con Brasil: Rocha, Treinta y tres, Cerro Largo, Rivera y Artigas. De ellos, Treinta y tres es el único que no posee frontera seca (terrestre) con Brasil y, por esta razón, no se incluyó en el ámbito de este Programa, realizado a lo largo de 2017 y 2018 en los departamentos de Cerro Largo, Rivera, Artigas y Rocha, del lado uruguayo, y en los municipios de Santana do Livramento y Aceguá (estado de Rio Grande do Sul), del lado brasileño (Figura 1).

\section{Planeación del Programa}

El Programa fue elaborado y ofertado por docentes de la ENSP/Fiocruz de Brasil, en cooperación con docentes de la Universidad de la República (UDELAR) de Uruguay. A través del trabajo continuo de un grupo coordinador que, además de representantes de las dos instituciones académicas, estaba compuesto por representantes del Ministerio de Salud de Brasil, del Ministerio de Salud Pública de Uruguay y de la Administración de los Servicios de Salud del Estado (ASSE) de Uruguay, se posibilitó el diseño, implementación y evaluación de un programa de formación de bajo costo, financiado con recursos propios y basado en la infraestructura existente de cada una de las instituciones mencionadas.

La estrategia de implementación, la perspectiva pedagógica y las habilidades y competencias a desarrollarse a lo largo del Programa se definieron en cuatro talleres de trabajo realizados entre agosto de 2014 y diciembre de 2016 con participación de aproximadamente 40 personas, entre profesores, actores de la práctica y gestores del sector salud de ambos países. En los dos primeros talleres se priorizó la identificación de demandas y prioridades locales en cada una de las zonas fronterizas previamente identificadas. A partir del mapeo y sistematización de la información producida se definieron las competencias y habilidades necesarias para el cumplimiento de los objetivos del Programa y, en los últimos dos talleres, estas se trasladaron en estrategias pedagógicas y contenidos específicos que definieron el itinerario formativo del Programa y las competencias por desarrollar.

\section{Perspectivas pedagógicas del Programa}

Una vez definido como objetivo principal del Programa el fortalecimiento de capacidades formativas locales, el diseño pedagógico privilegió la "formación de formadores"; estos profesionales-formadores deberían actuar, al final del Programa y de manera permanente, como activadores de procesos educativos junto a sus equipos de trabajo, bajo la perspectiva de la educación permanente en salud $(17,19)$. 
FIGURA 1. Mapa de la Frontera Brasil Uruguay con identificación de los locales de realización de las actividades del Programa de Formación: Montevideo (1), Rocha (2), Melo (3), Artigas (4), Rivera y Santana do Livramento (5) y Aceguá (6).

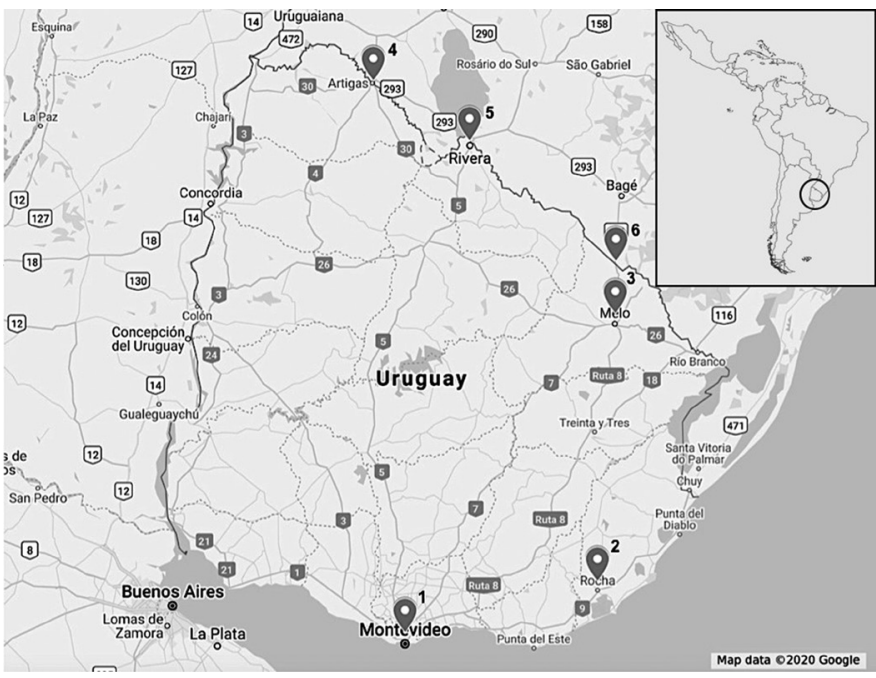

Fuente: elaboración propia a partir de mapa base público de @Google Maps Nota: Las denominaciones empleadas y la forma en que aparecen presentados los datos que contiene no implican, por parte de la OPS o la RPSP, juicio alguno sobre la condición jurídica de países, territorios, ciudades o zonas, o de sus autoridades, ni respecto del trazado de sus fronteras o límites.

Las actividades del Programa se estructuraron a partir de talleres pedagógicos ofrecidos en los municipios fronterizos por docentes brasileños y uruguayos para profesionales que trabajaban o coordinaban servicios de salud en el ámbito de la atención primaria y de otros niveles de organización en ambos países. Estos profesionales se identificaron y seleccionaron con base en su experiencia en acciones de capacitación de equipos de salud y su capacidad de liderar procesos de cambio en los servicios de salud de cada zona fronteriza. Se identificó a 35 profesionales que cumplían las condiciones definidas, de los cuales 27 (23 uruguayos y 4 brasileños) permanecieron durante todo el Programa y fueron certificados al final del primer año.

Las estrategias pedagógicas se basaron en metodologías activas de la educación en salud $(17,20)$ donde los docentes actuaron como mediadores del proceso de reconocimiento de los desafíos y oportunidades presentes en el trabajo cotidiano y locoregional. A partir de los ejemplos y contribuciones aportados por los profesionales-alumnos, los docentes presentaban los marcos conceptuales aplicables y abordajes metodológicos/herramientas de diagnóstico y análisis adecuados a cada situación, teniendo en cuenta las diferencias políticas y en los sistemas y servicios de salud en ambos países.

\section{Etapas del Programa}

Las actividades del Programa se estructuraron a lo largo de dos años. El primero de ellos se dedicó a la formación de formadores $\mathrm{y}$, el segundo, al acompañamiento de la actuación de los profesionales-alumnos como formadores junto a sus equipos.

En el primer año, el programa académico se organizó en tres Unidades de Aprendizaje (Cuadro 1), durante las cuales se utilizaron metodologías activas de la educación en salud (p. ej., como dramatizaciones, estudios de casos, problematización de situaciones reales) que tuvieron como objetivo retratar el
CUADRO 1. Programa de curso del primer año del Programa de Formación, Uruguay, 2017

\begin{tabular}{|c|c|c|c|}
\hline \multicolumn{4}{|c|}{2017} \\
\hline & \multicolumn{3}{|c|}{ UA 1 - Atención Integral, Políticas y Gestión en Salud } \\
\hline & Tema & $\begin{array}{l}\text { Abordaje } \\
\text { Pedagógico }\end{array}$ & Lugar y Fecha \\
\hline Módulo 1 & $\begin{array}{l}\text { Reconocimiento del } \\
\text { territorio y de las } \\
\text { características de los } \\
\text { sistemas de salud local }\end{array}$ & $\begin{array}{l}\text { Problematización } \\
\text { Dramatización }\end{array}$ & $\begin{array}{c}\text { Montevideo } \\
\text { (Montevideo), marzo } \\
\text { de } 2017\end{array}$ \\
\hline Módulo 2 & $\begin{array}{l}\text { Organización y gestión } \\
\text { de la Atención Integral } \\
\text { a la Salud en la frontera } \\
\text { y las redes de atención } \\
\text { en salud }\end{array}$ & $\begin{array}{l}\text { Problematización } \\
\text { Dramatización } \\
\text { Estudio Dirigido }\end{array}$ & $\begin{array}{c}\text { Rocha (Rocha), abril } \\
\text { de } 2017\end{array}$ \\
\hline Módulo 3 & $\begin{array}{l}\text { Organización de los } \\
\text { programas de salud y } \\
\text { la centralización de la } \\
\text { Atención Primaria en } \\
\text { Salud }\end{array}$ & $\begin{array}{l}\text { Problematización } \\
\text { Estudio Dirigido }\end{array}$ & $\begin{array}{l}\text { Rocha (Rocha), } \\
\text { mayo de } 2017\end{array}$ \\
\hline Módulo 4 & $\begin{array}{l}\text { Métodos e instrumentos } \\
\text { de planeamiento y } \\
\text { gestión en salud }\end{array}$ & $\begin{array}{l}\text { Problematización } \\
\text { Dramatización }\end{array}$ & $\begin{array}{l}\text { Melo (Cerro Largo), } \\
\text { junio de } 2017\end{array}$ \\
\hline \multicolumn{4}{|c|}{ UA 2 - Vigilancia en Salud } \\
\hline Módulo 5 & $\begin{array}{l}\text { Marcos Teórico- } \\
\text { conceptuales de } \\
\text { Vigilancia en Salud y los } \\
\text { sistemas de información }\end{array}$ & $\begin{array}{l}\text { Problematización } \\
\text { Estudio Dirigido }\end{array}$ & $\begin{array}{l}\text { Melo (Cerro Largo), } \\
\text { julio de } 2017\end{array}$ \\
\hline Módulo 6 & $\begin{array}{l}\text { Manejo de bases de } \\
\text { datos }\end{array}$ & $\begin{array}{c}\text { Análisis de } \\
\text { banco de datos }\end{array}$ & $\begin{array}{l}\text { Artigas (Artigas), } \\
\text { agosto de } 2017\end{array}$ \\
\hline Módulo 7 & $\begin{array}{c}\text { Investigación } \\
\text { epidemiológica y la } \\
\text { evaluación de las } \\
\text { acciones de vigilancia } \\
\text { en salud en la frontera }\end{array}$ & $\begin{array}{l}\text { Problematización } \\
\text { Estudio Dirigido }\end{array}$ & $\begin{array}{l}\text { Artigas (Artigas), } \\
\text { setiembre de } 2017\end{array}$ \\
\hline \multicolumn{4}{|c|}{ UA 3 - Educación y Promoción de la Salud } \\
\hline Módulo 8 & $\begin{array}{l}\text { La Educación en Salud y } \\
\text { los diferentes modelos } \\
\text { formativos aplicados } \\
\text { a la formación en } \\
\text { servicios }\end{array}$ & $\begin{array}{l}\text { Problematización } \\
\text { Estudio Dirigido }\end{array}$ & $\begin{array}{l}\text { Rivera (Rivera), } \\
\text { octubre de } 2017\end{array}$ \\
\hline Módulo 9 & $\begin{array}{l}\text { Promoción de la salud y } \\
\text { su papel en la formación } \\
\text { de trabajadores y en } \\
\text { el enfrentamiento de } \\
\text { problemas de salud en la } \\
\text { frontera }\end{array}$ & $\begin{array}{l}\text { Problematización } \\
\text { Estudio Dirigido }\end{array}$ & $\begin{array}{l}\text { Rivera (Rivera), } \\
\text { noviembre de } 2017\end{array}$ \\
\hline Módulo 10 & $\begin{array}{l}\text { La construcción, } \\
\text { desarrollo y evaluación } \\
\text { de una acción formativa } \\
\text { en salud }\end{array}$ & $\begin{array}{l}\text { Problematización } \\
\text { Estudio Dirigido }\end{array}$ & $\begin{array}{c}\text { Montevideo } \\
\text { (Montevideo), } \\
\text { diciembre de 2017 }\end{array}$ \\
\hline
\end{tabular}

Fuente: elaboración propia.

trabajo cotidiano y señalar la posibilidad de incorporar nuevas herramientas y conocimientos (20).

Las tres Unidades de Aprendizaje se diseñaron de manera que respondieran a los objetivos específicos del Programa. La Unidad de Aprendizaje 1 (Atención Integral, Políticas y Gestión en Salud) posibilitó a los profesionales-alumnos conocer, en una perspectiva comparada, las especificidades de cada sistema de salud (brasileño y uruguayo), a partir de ejemplos y situaciones-problema presentadas por estos profesionales, debidamente contextualizados por aportes teóricos y conceptuales de los profesores de la ENSP/Fiocruz y la UDELAR. 
La Unidad de Aprendizaje 2 (Vigilancia en Salud) posibilitó el conocimiento y la aplicación de herramientas y principios de la epidemiología y de la estadística para el análisis de datos y situaciones de salud. A partir del acceso a bases de datos nacionales de ambos países, los profesionales-alumnos tuvieron la oportunidad de realizar análisis de situaciones-problema de interés local e interpretar los datos para definir prioridades en atención, vigilancia y promoción de la salud.

Por fin, la Unidad de Aprendizaje 3 (Educación y Promoción de la Salud) posibilitó la discusión y aplicación práctica de marcos teóricos y metodológicos de la educación en salud y de la promoción de la salud en el diseño de una estrategia formativa para los equipos de salud de cada zona fronteriza, proyecto este que se configuró como Trabajo de Conclusión de la primera etapa del Programa.

Cada módulo preveía 16 horas de actividades presenciales y 8 horas de actividad en el ambiente virtual de aprendizaje (plataforma Moodle configurada específicamente para el programa), con un total de 24 horas de actividades didácticas por mes, durante 10 meses (de marzo a diciembre de 2017).

En el segundo año del programa, desarrollado entre agosto y noviembre de 2018, los profesionales-alumnos egresados de la primera etapa del Programa $(n=27)$ tuvieron la oportunidad de poner en práctica los proyectos de formación que habían presentado en la última Unidad de Aprendizaje. Cada región fronteriza definió sus prioridades y diseñó una acción formativa con base en estas prioridades y siguiendo las premisas trabajadas durante el primer año (Cuadro 2).

\section{RESULTADOS}

Participaron de las dos etapas del Programa 27 profesionales-alumnos, 23 uruguayos y cuatro brasileños (25 mujeres y 2 varones). En cuanto a la formación de base, todos tenían nivel superior completo (19 licenciados en enfermería, 3 médicos, 3 licenciados en trabajo social y 2 psicólogos).

Con relación a la inserción en los servicios y programas de salud, 22 profesionales-alumnos actuaban en equipos de atención primaria en salud y 5 en la gestión de programas y servicios de salud. En cuanto a la vinculación institucional, 19 eran trabajadores de ASSE/Uruguay, 4 del Ministerio de Salud Pública de Uruguay y 4 estabam vinculados a Secretarías Municipales de Salud de Aceguá y Santana do Livramento, en Rio Grande do Sul, Brasil.

Solamente en dos zonas fronterizas participaron profesionales-alumnos de ambos países: en Rivera-Santana do Livramento y en Cerro Largo-Aceguá. En las zonas de Rocha-Chuí y Artigas-Quaraí solamente participaron del Programa profesionales uruguayos (departamentos de Rocha y Artigas).

Los principales resultados del Programa están relacionados al fortalecimiento de capacidades locales en salud -en particular las capacidades de formación y capacitación de equipos en salud pública- y al fortalecimiento de capacidades de investigación y análisis para la vigilancia en salud.

\section{Fortalecimiento de capacidades formativas en la frontera}

En primer año del Programa (2017) se organizó cuatro equipos de trabajo multidisciplinarios dedicados a la formación de trabajadores de salud en las cuatro zonas fronterizas
CUADRO 2. Actividades desarrolladas en el segundo año del Programa de Formación, Uruguay, 2018

\begin{tabular}{|c|c|c|c|}
\hline \multicolumn{4}{|c|}{2018} \\
\hline \multicolumn{4}{|c|}{ Departamento de Cerro Largo - Municipio de Aceguá } \\
\hline & $\begin{array}{l}\text { Tema del Proyecto } \\
\text { Formativo }\end{array}$ & $\begin{array}{l}\text { Estrategia } \\
\text { Formativa }\end{array}$ & Lugar y Fecha \\
\hline $\begin{array}{l}\text { Proyecto de } \\
\text { Formación }\end{array}$ & $\begin{array}{l}\text { Organización de un } \\
\text { Grupo Binacional } \\
\text { de Salud para a la } \\
\text { Frontera }\end{array}$ & $\begin{array}{c}\text { Reuniones } \\
\text { Visitas Técnicas }\end{array}$ & $\begin{array}{l}\text { Melo (Cerro Largo/ } \\
\text { UY) y Aceguá (RS/ } \\
\text { BR), de junio de } \\
2017 \text { a agosto de } \\
2018\end{array}$ \\
\hline $\begin{array}{l}\text { Actividad de } \\
\text { Culminación }\end{array}$ & $\begin{array}{l}\text { Feria Binacional de } \\
\text { Salud }\end{array}$ & $\begin{array}{l}\text { Feria de Salud } \\
\text { Visita a las } \\
\text { Unidades de Salud }\end{array}$ & $\begin{array}{l}\text { Aceguá (Cerro Largo/ } \\
\text { UY), agosto de } 2018\end{array}$ \\
\hline \multicolumn{4}{|c|}{ Departamento de Rivera - Municipio de Santana do Livramento } \\
\hline & $\begin{array}{c}\text { Tema del Proyecto } \\
\text { Formativo }\end{array}$ & $\begin{array}{l}\text { Estrategia } \\
\text { Formativa }\end{array}$ & Lugar y Fecha \\
\hline $\begin{array}{l}\text { Proyecto de } \\
\text { Formación }\end{array}$ & $\begin{array}{l}\text { Organización de un } \\
\text { Grupo Binacional de } \\
\text { Salud para la Frontera }\end{array}$ & $\begin{array}{c}\text { Reuniones } \\
\text { Visitas Técnicas }\end{array}$ & $\begin{array}{c}\text { Rivera (Rivera/ } \\
\text { UY) y Sant'Ana do } \\
\text { Livramento (RS/BR), } \\
\text { de junio de } 2017 \text { a } \\
\text { setiembre de } 2018\end{array}$ \\
\hline $\begin{array}{l}\text { Actividad de } \\
\text { Culminación }\end{array}$ & $\begin{array}{c}\text { Vigilancia en Salud } \\
\text { sobre Enfermedades } \\
\text { de transmisión sexual } \\
\text { en Masoller }\end{array}$ & $\begin{array}{l}\text { Visita Técnica } \\
\text { Paneles } \\
\text { Acción de Salud } \\
\text { en Campo }\end{array}$ & $\begin{array}{l}\text { Masoller (Rivera/ } \\
\text { UY), setiembre de } \\
2018\end{array}$ \\
\hline \multicolumn{4}{|c|}{ Departamento de Rocha } \\
\hline & $\begin{array}{l}\text { Tema del Proyecto } \\
\text { Formativo }\end{array}$ & $\begin{array}{l}\text { Estrategia } \\
\text { Formativa }\end{array}$ & Lugar y Fecha \\
\hline $\begin{array}{l}\text { Proyecto de } \\
\text { Formación }\end{array}$ & $\begin{array}{l}\text { Curso Territorio y } \\
\text { Población (ASSE) }\end{array}$ & $\begin{array}{l}\text { Curso de } \\
\text { Formación } \\
\text { Profesional }\end{array}$ & $\begin{array}{l}\text { Rocha, de junio a } \\
\text { setiembre de } 2018\end{array}$ \\
\hline $\begin{array}{l}\text { Actividad de } \\
\text { Culminación }\end{array}$ & $\begin{array}{l}\text { Mesa Redonda Sobre } \\
\text { Ios Desafíos de la } \\
\text { Organización de la } \\
\text { Atención Primaria en } \\
\text { Salud }\end{array}$ & $\begin{array}{l}\text { Mesa Redonda } \\
\text { Circulo de } \\
\text { conversación }\end{array}$ & $\begin{array}{c}\text { Rocha, octubre de } \\
2018\end{array}$ \\
\hline \multicolumn{4}{|c|}{ Departamento de Artigas } \\
\hline & $\begin{array}{l}\text { Tema del Proyecto } \\
\text { Formativo }\end{array}$ & $\begin{array}{l}\text { Estrategia } \\
\text { Formativa }\end{array}$ & Lugar y Fecha \\
\hline $\begin{array}{l}\text { Proyecto de } \\
\text { Formación }\end{array}$ & $\begin{array}{l}\text { Curso Territorio y } \\
\text { Población (ASSE) }\end{array}$ & $\begin{array}{l}\text { Curso de } \\
\text { Formación } \\
\text { Profesional }\end{array}$ & $\begin{array}{l}\text { Artigas, de junio a } \\
\text { setiembre de } 2018\end{array}$ \\
\hline $\begin{array}{l}\text { Actividad de } \\
\text { Culminación }\end{array}$ & $\begin{array}{l}\text { Conferencias Sobre } \\
\text { Políticas de Atención } \\
\text { Primaria en Uruguay y } \\
\text { Educación Permanente } \\
\text { en Salud }\end{array}$ & Conferencias & $\begin{array}{c}\text { Artigas, noviembre } \\
\text { de } 2018\end{array}$ \\
\hline
\end{tabular}

Fuente: elaboración propia.

participantes; dos equipos eran binacionales (Artigas-Santana do Livramento y Cerro Largo-Aceguá) y dos nacionales (Rocha y Artigas, ambos uruguayos). Estos equipos asumieron, en sus respectivos departamentos y municipios, los programas de formación y educación permanente de equipos de atención primaria en salud, tornándose referentes para diferentes proyectos y programas de desarrollo de recursos humanos de ASSE y del Ministerio de Salud Pública.

Entre las acciones coordinadas por los equipos del Programa se destaca la coordinación y el trabajo docente en el curso “Territorio y Población: abordaje y gestión desde los equipos básicos" de ASSE en los departamentos de Rocha y Artigas. Este curso se centró en conformar Equipos Básicos en Territorio 
(EBT) capacitados para brindar atención integral en salud a la población a cargo. En 2018 fueron capacitados cerca de 20 profesionales en cada uno de estos dos departamentos fronterizos por los profesionales-alumnos que concluyeron la primera etapa, bajo la supervisión del equipo coordinador y apoyo académico de profesores de la ENSP/Fiocruz y la UDELAR.

Asimismo, en la zona fronteriza de Rivera-Santana do Livramento, el equipo binacional organizó un programa de capacitación de trabajadores de salud y líderes comunitarios de las localidades de Massoller (Uruguay) y Vila Tomáz Albornoz (Brasil) dedicado a la organización de la atención y de la vigilancia del VIH/sida en esta zona fronteriza, caracterizada por la actividad ganadera y con antecedentes extensos de casos de enfermedades de transmisión sexual asociados a la prostitución.

En las cuatro zonas fronterizas, los equipos multidisciplinarios formados por el Programa asumieron las acciones de formación y capacitación en el nivel local y organizaron una agenda permanente de desarrollo de recursos humanos en salud, todavía en curso.

\section{Fortalecimiento de capacidades de vigilancia en salud en la frontera}

En la zona fronteriza de Cerro Largo-Aceguá, el equipo binacional organizó una agenda de trabajo continua, con encuentros mensuales, dedicada a la discusión y monitoreo de situaciones de salud a ambos lados de la frontera. A partir del conocimiento sobre vigilancia en salud desarrollado durante el primer año del Programa, los profesionales-alumnos egresados realizaron un conjunto de análisis de situaciones de salud y búsqueda activa de casos de diferentes enfermedades, elaborando mapas y gráficos comparativos como elementos clave para la toma de decisión por las autoridades de ambos países.

Igualmente, en la zona de Rivera-Santana do Livramento, el trabajo del equipo binacional posibilitó la organización de una agenda de trabajo conjunta, creando sinergias y convergencias entre los distintos programas ofrecidos a ambos lados de la frontera, optimizando recursos y estableciendo complementariedades para la atención y la promoción de la salud. Entre las acciones conjuntas se destaca el programa de inmunización local (con calendarios ajustados en los dos lados de la frontera) y los análisis conjuntos de la vigilancia de las arbovirosis (dengue, zika y chikungunya).

\section{CONTRIBUCIONES DEL PROGRAMA A LA ATENCIÓN Y VIGILANCIA EN SALUD EN LA FRONTERA}

La estrategia de organización durante el primer año del Programa, junto con la articulación interinstitucional establecida por el equipo coordinador, permitió que las acciones planificadas por los profesionales-alumnos a lo largo del curso se incorporaran a la estructura de los servicios y programas de salud locales, dando institucionalidad y continuidad a los proyectos desarrollados durante la capacitación. A través de una estrategia de seguimiento de las actividades por el equipo coordinador, basadas en la evaluación formativa del Programa, fue posible observar un conjunto de contribuciones a los servicios, programas y políticas de salud en las zonas fronterizas participantes.
Un aspecto a destacar es el diseño de estrategia formativa binacional basada en metodologías activas y centrada en la estrategia de "formador de formadores". A partir del trabajo organizado a lo largo de dos años por los cuatro equipos multidisciplinarios fue posible extender la experiencia formativa inicial, de manera continua, a otros equipos de salud y a la comunidad. La conceptualización de educación permanente en salud ha sido un aporte sustancial del Programa a los servicios de salud, con incorporación en las tareas cotidianas de los equipos de salud.

La articulación entre los equipos multidisciplinarios de cada una de las cuatro zonas fronterizas con referentes académicos de Brasil y Uruguay también posibilitó el desarrollo de competencias específicas para el análisis de situaciones de salud en el nivel local, la investigación epidemiológica y la vigilancia en salud de base territorial, estrategias que fueron incorporadas a los programas y servicios de atención y vigilancia en salud de las cuatro zonas fronterizas, donde no existían tales competencias antes del Programa.

Esta articulación este los actores de la práctica y los referentes académicos posibilitó el aporte, por parte de docentes y profesionales-alumnos egresados del Programa, de una contribución al Informe Final de la I Conferencia Nacional de Salud de Brasil en 2019 sobre el tema de la vigilancia de fronteras, que anteriormente no se había considerado.

Teniendo en cuenta las demandas de formación en Uruguay y la escasez de cursos más avanzados en epidemiología, se discutió y aprobó en el marco de la cooperación entre la ENSP/ Fiocruz y la UDELAR la oferta del Curso de Maestría Profesional en Epidemiología Aplicada a los Servicios de Salud de la ENSP/Fiocruz, enfocada en la calificación de docentes de la UDELAR (Cuadro 3). Esta se inició en octubre de 2018 y se estima su finalización en diciembre de 2020. Los profesionales egresados de la maestría se constituirán en referentes nacionales, incluso para los equipos multidisciplinarios de las cuatro zonas fronterizas, y apoyarán el análisis de situaciones de salud y la vigilancia en salud de base territorial.

La principal limitación del Programa ha sido la imposibilidad de contar con equipos binacionales en dos de las cuatro zonas fronterizas, lo que impidió el alcance pleno de los objetivos planteados. Además, la ausencia de un reglamento binacional específico para la atención y vigilancia de la salud en la frontera Brasil-Uruguay dificultó la formalización de acciones conjuntas estructuradas, realizadas hasta el momento bajo acuerdos informales entre gestores de ambos los lados de la frontera, lo que representa un marco frágil cuando se considera los cambios periódicos de gobierno en los municipios, departamentos fronterizos y los niveles federales de Brasil y Uruguay.

\section{CONCLUSIONES}

El diseño metodológico, las competencias definidas y la gestión basados en un equipo coordinador binacional del Programa posibilitaron el desarrollo de capacidades formativas y de investigación junto a un grupo de profesionales de cuatro zonas fronterizas, y la constitución de equipos multidisciplinarios (dos binacionales y dos nacionales) de referencia para la formación y calificación de recursos humanos en salud y para el análisis de situaciones de salud en el marco de la vigilancia en salud de Brasil y Uruguay. 
CUADRO 3. Programa del Curso de Maestría Profesional en Epidemiologia Aplicada a los Servicios de Salud, Uruguay, 2018

\begin{tabular}{|c|c|c|c|}
\hline Disciplinas & Fecha & $\begin{array}{l}\text { Carga Horaria } \\
\text { (horas) }\end{array}$ & Créditos \\
\hline $\begin{array}{l}\text { Notas Introductorias a } \\
\text { la Epidemiología y a la } \\
\text { Bioestadística }\end{array}$ & Octubre de 2018 & 45 & 3 \\
\hline Epidemiología I & Noviembre de 2018 & 45 & 3 \\
\hline Seminarios Avanzados I & Diciembre de 2018 & 45 & 3 \\
\hline $\begin{array}{l}\text { Actividades de Orientación } \\
\text { a distancia }\end{array}$ & Enero de 2019 & - & - \\
\hline $\begin{array}{l}\text { Actividades de Orientación } \\
\text { a distancia }\end{array}$ & Febrero de 2019 & - & - \\
\hline $\begin{array}{l}\text { Estadística Aplicada a } \\
\text { Epidemiología }\end{array}$ & Marzo de 2019 & 45 & 3 \\
\hline $\begin{array}{l}\text { Encuestas } \\
\text { Epidemiológicas }\end{array}$ & Abril de 2019 & 45 & 3 \\
\hline Epidemiología II & Mayo de 2019 & 45 & 3 \\
\hline Modelos Estadísticos I & Junio de 2019 & 45 & 3 \\
\hline Seminarios Avanzados II & Julio de 2019 & 45 & 3 \\
\hline Epidemiología III & Agosto de 2019 & 45 & 3 \\
\hline Modelos Estadísticos II & Setiembre de 2019 & 45 & 3 \\
\hline $\begin{array}{l}\text { Seminarios Avanzados III } \\
\text { (Pre-cualificación) }\end{array}$ & Octubre de 2019 & 45 & 3 \\
\hline $\begin{array}{l}\text { Cualificación de los } \\
\text { Proyectos }\end{array}$ & Noviembre de 2019 & - & - \\
\hline $\begin{array}{l}\text { Seminarios Avanzados IV } \\
\text { (Pre-defensa) }\end{array}$ & Septiembre de 2020 & 45 & 3 \\
\hline $\begin{array}{l}\text { Defensas de las } \\
\text { disertaciones }\end{array}$ & Diciembre de 2020 & - & - \\
\hline
\end{tabular}

El acercamiento y mayor diálogo entre los equipos formados y otros actores de la práctica, gestores, académicos y líderes locales posibilitó la organización de una agenda permanente de formación e investigación, con énfasis en el análisis comparado de las políticas, los programas y los sistemas de salud de ambos los países. Asimismo, posibilitó la aplicación de técnicas y herramientas de análisis de datos de salud en los niveles local y regional por grupos binacionales constituidos a lo largo del Programa. El diseño del Programa y las competencias desarrolladas a lo largo de dos años posibilitaron el desarrollo en el nivel local de estrategias formativas basadas en metodologías activas de la educación permanente en salud, lo que contribuyó de manera efectiva al fortalecimiento de capacidades formativas y de investigación en la frontera Brasil-Uruguay.

Los logros del Programa, la positiva relación costo-efectividad de la acción como un todo y sus contribuciones a la organización de los servicios de salud locales lo califican como una acción estratégica para el fortalecimiento de capacidades formativas y de investigación en las cuatro zonas fronterizas participantes. Se recomienda el desarrollo y la implementación de iniciativas similares en otros países de América Latina cuyas fronteras presenten características similares a la frontera entre Brasil y Uruguay.

Contribución de los autores. Todos los autores concibieron y planificaron el estudio original, recolectaron, analizaron e interpretaron los datos, escribieron y revisaron el manuscrito. Todos los autores revisaron y aprobaron la versión final.

Agradecimientos. Los autores agradecen al Ministerio de Salud Pública, a la Administradora de los Servicios de Salud del Estado, a la Universidad de la República, al Consejo de Educación Técnico Profesional y a las Direcciones Departamentales de Salud de Rocha, Cerro Largo, Artigas y Rivera, en Uruguay, así como al Ministerio de Salud, a la Escuela Nacional de Salud Pública Sergio Arouca, a la Fundación Oswaldo Cruz, a la Secretaría de Salud del Estado de Rio Grande do Sul y a las Secretarías Municipales de Salud de Aceguá y Santana do Livramento, en Brasil, por todo el apoyo logístico y financiero, indispensable a la realización del Programa de Formación.

Conflictos de intereses. Ninguno declarado.

Declaración. Las opiniones expresadas en este manuscrito son responsabilidad del autor y no reflejan necesariamente los criterios ni la política de la RPSP/PAJPH y/o de la OPS.

\section{REFERENCIAS}

1. Rovere MR. Planificación estratégica de recursos humanos en salud. 2a Ed. Washington DC: OPS; 2006.

2. Portela GZ, Fehn AC, Ungerer RLS, Dal Poz MR. Human resources for health: global crisis and international cooperation. Ciênc. saúde coletiva. 2017; 22 (7):2237-46.

3. Organizacíon Mundial de la Salud. Working for health and growth: investing in the health workforce. Report of the High-Level Commission on Health Employment and Economic Growth. Ginebra: OMS; 2016.

4. Organización Panamericana de la Salud. Estrategia de Recursos Humanos para el Acceso Universal a la Salud y la Cobertura Universal de Salud. 29a Conferencia Panamericana de Salud, Comité Regional de la OMS para las Américas [Internet]. Washington DC: OPS; 2017 [citado 2020 jun 17]. Disponible en: https://www. observatoriorh.org/sites/default/files/webfiles/fulltext/2017/ CSP29-10-s.pdf

5. Organización Mundial de la Salud. Estrategia mundial de recursos humanos para la salud: personal sanitario 2030. $69^{\mathrm{a}}$ Asamblea Mundial de la Salud [Internet]. Ginebra: OMS; 2016 [citado 2020 jun 17]. Disponible en: http://apps.who.int/gb/ebwha/pdf_files/ WHA69/A69_R19-sp.pdf
6. Giovanella L, Almeida PF. Atenção primária integral e sistemas segmentados de saúde na América do Sul. Cad. Saúde Pública [Internet]. 2017 [citado 2020 Jun 17]; 33 (Suppl 2): e00118816. Disponible en: http://www.scielo.br/scielo.php?script=sci_arttext\&pid=S0102-311X2017001405005\&lng=en. Epub Oct 02, 2017. http:/ / dx.doi.org/10.1590/0102-311x00118816.

7. Giovanella L, Guimarães L, Nogueira VMR, Lobato LVC, Damacena GN. Saúde nas fronteiras: acesso e demandas de estrangeiros e brasileiros não residentes ao SUS nas cidades de fronteira com países do MERCOSUL na perspectiva dos secretários municipais de saúde. Cad Saúde Pública. 2007;23 (suppl 2):S251-66.

8. Schweiger ALF, Álvarez DT. Integración regional, necesidades de salud de la población y dotación de recursos humanos en sistemas y servicios de salud: aproximación al concepto de estimación de brecha. Cad Saúde Pública. 2007; 23:S202-S213.

9. Fagundes HS, Kreutz IT, Nogueira VMR, Castamann D. Health on the Brazil-Uruguay border: pacts and protagonism of local actors. Revista Katálysis. 2018; 21(2):293-304.

10. Clemente I. La región de frontera Uruguay-Brasil y la relación binacional: pasado y perspectivas. Rev Urug Cienc Polít. 2010; 19:165-84. 
11. Lemões MAM, Lange C, Castro DSP, Llano PMP, Machado RA, Hermes GB. Programa más médicos en la frontera: gestión en salud en las ciudades gemelas entre Brasil y Uruguay. Rev Urug Enferm. 2019;14 (1):38-48.

12. Rótulo D, Baudean M, Abrucio F, Cohn N. Gobernanza y políticas de cooperación fronteriza: el caso Brasil-Uruguay. Revista del CLAD Reforma y Democracia. 2014; 58:135-66.

13. Pucci AS. O estatuto da fronteira Brasil-Uruguai [Internet]. Brasília: Fundação Alexandre de Gusmão; 2010. [citado 2020 jun 17]. Disponible en: http://funag.gov.br/biblioteca/download/685-O_ estatuto_da_fronteira_brasil_uruguai.pdf

14. Giovanella L, Guimarães L, Damacena GN, Teixeira CP, de Almeida PF, Prá KRD, et al. Saúde nas fronteiras: estudo do acesso aos serviços de saúde nas cidades de fronteira com países do Mercosul [Internet]. Rio de Janeiro: ENSP/Fiocruz; 2007 [citado 2020 jun 17]. Disponible en: http://www.ensp.fiocruz.br/portal-ensp/saudefronteiras/pdf/pesquisa_divulgacao_01.pdf

15. Bentura C, Ortega E. Salud en la frontera Uruguay Brasil. Un estudio exploratoriodelalegislación uruguaya.XIIIJornadasdeInvestigación de la Facultad de Ciencias Sociales [Internet]. Montevideo: UDELAR; 2014 [citado 2020 jun 17]. Disponible en: https://pdfs.semanticscholar.org/ba94/cedf450890023fdd9e28b7a1f70ef01abe1a. pdf?_ga=2.219922137.1373978210.1592401887-2117471356.1532721530

16. Guimarães L, Giovanella L. Municípios brasileiros fronteriços e Mercosul: características e iniciativas de cooperação em saúde. Saúde e debate. 2005;29(71):248-57.
17. Merhy EE, Feuerwerker LCM, Ceccim RB. Educación permanente en salud: una estrategia para intervenir en la micropolítica del trabajo en salud. Salud colectiva. 2006; 2:147-60.

18. Castro-Jiménez LE, Rincón-Moreno M, Gómez-Rodríguez DT. Educación para la salud: una mirada desde la antropología. Rev Ciencias de la Salud. 2017; 15 (1):145-63.

19. Gigante RL, Campos GWDS. Política de Formação e Educação Permanente em Saúde no Brasil: bases legais e referências teóricas. Trabalho, Educação e Saúde. 2016; 14 (3):747-63.

20. Farias PAM, Martin ALDAR, Cristo CS. Aprendizagem ativa na educação em saúde: percurso histórico e aplicações. Rev Bras Educ Méd. 2015;39(1):143-50.

21. França RG. Saúde na fronteira Brasil-Uruguai e o Projeto de Cooperação Trilateral Brasil-Alemanha-Uruguai. En: AISA. Saúde e Política Externa: os 20 anos da Assessoria de Assuntos Internacionais de Saúde (1998-2018) [Internet]. Brasília: Ministério da Saúde; 2018 [citado 2020 jun 17]. Disponible en: http://www.saude.gov. br/images/pdf/2018/outubro/22/14_Rafael_Franca.pdf

Manuscrito recibido el 15 de enero de 2020. Aceptado para su publicación, tras revisión, el 24 de junio de 2020.

\section{Public Health Training Program for Health Workers on the Brazil-Uruguay Border}

ABSTRACT The main objective of the Public Health Training Program on the Brazil-Uruguay Border was to strengthen local training capacities in public health through the development of skills and competencies together with health workers from four border areas. The methodological design of the Program, based on workshops with the application of active methodologies in health training and mediated by educational technologies, favored the organization of a permanent dialogical setting between instructors, managers and practice professionals from both countries that, between 2017 and 2018, resulted in a) development of local capacities for the mapping of the main health surveillance problems in the four participating border areas; $b$ ) discussion of the limits and perspectives of action of the existing health services in both countries, within the principles and guidelines of the public health field; c) appropriation of pedagogical tools and processes that allowed for the incorporation of public health and health surveillance into the different organizational processes and settings of the local health sector; and d) building of a permanent capacity for the development of skills and competencies together with health workers, within a framework of permanent education in health. The Program's achievements and its contributions to local health services effectively qualify it as a strategic action for strengthening training and research capacities on the Brazil-Uruguay border.

Keywords $\quad$ Staff development; problem-based learning; education, public health professional; public health surveillance; border health; Brazil; Uruguay. 